Pacific Journal of Mathematics

CHEBYSHEV APPROXIMATION TO ZERO

JAMES MCLEAN SLOBS 


\section{CHEBYSHEV APPROXIMATION TO ZERO}

\section{JAMES M. SLOSS}

In this paper we shall be concerned with the questions of existence, uniqueness and constructability of those polynomials in $k+1$ variables $\left(x_{1}, x_{2}, \cdots, x_{k}, y\right)$ of degree not greater than $n_{s}$ in $x_{s}$ and $m$ in $y$ which best approximate zero on $I_{1} \times I_{2} \times \cdots \times I_{k+1}, I_{s}=[-1,1]$, in the Chebyshev sense.

It is a classic result that among all monic polynomials of degree not greater than $n$ there is a unique polynomial whose maximum over the interval $[-1,1]$ is less than the maximum over $[-1,1]$ of any other polynomial of the same type and moreover it is given by $\widetilde{T}_{n}(x)=$ $2^{1-n} \cos [n$ are $\cos x]$, the normalized Chebyshev polynomial.

Our method of attack will be to prove a generalization of an inequality for monic polynomials in one variable concerning the lower bound of the maximum viz. $\max _{-1 \leqq x \leqq 1}\left|P_{n}(x)\right| \geqq 2^{1-n}$ where $P_{n}(x)$ is a monic polynomial of degree not greater than $n$. The theorem will show that the only hope for uniqueness is to normalize our class of polynomials. This is done in a very natural way viz. by considering only polynomials, if they exist, of the form:

$$
\begin{aligned}
P\left(x_{1}, x_{2}, \cdots, x_{k}, y\right)= & A_{m}\left(x_{1}, \cdots, x_{k}\right) y^{m} \\
& +A_{m-1}(\cdots) y^{m-1}+\cdots+A_{0}(\cdots)
\end{aligned}
$$

for which $A_{m}\left(x_{1}, x_{2}, \cdots, x_{k}\right)$ is the best polynomial approximation to zero on $I_{1} \times I_{2} \times \cdots \times I_{k}$. Thus if $k=1$, we consider only polynomials of the form:

$$
P\left(x_{1}, y\right)=\widetilde{T}_{n}\left(x_{1}\right) y^{m}+A_{m-1}\left(x_{1}\right) y^{m-1}+\cdots+A_{0}\left(x_{1}\right) .
$$

We find in the case of $(0.2)$ that there is a unique best polynomial approximation and it is given by $\widetilde{T}_{n}\left(x_{1}\right) \widetilde{T}_{m}(y)$. Thus we can consider the question of existence, uniqueness and constructability of a polynomial of the form:

$$
\begin{aligned}
P\left(x_{1}, x_{2}, y\right)= & \widetilde{T}_{n_{1}}\left(x_{1}\right) \widetilde{T}_{n_{2}}\left(x_{2}\right) y^{m} \\
& +A_{m-1}\left(x_{1}, x_{2}\right) y^{m-1}+\cdots+A_{0}\left(x_{1}, x_{2}\right)
\end{aligned}
$$

that best approximates zero. We find in this case there is a unique best polynomial approximation and it is given by $\widetilde{T}_{n_{1}}\left(x_{1}\right) \widetilde{T}_{n_{2}}\left(x_{2}\right) \widetilde{T}_{m}(y)$. Continuing in this way we shall show that the question is meaning-

Received December 18, 1963. 
ful in general and that there is a unique best polynomial approximation to zero of the form (0.1) given by $\widetilde{T}_{n_{1}}\left(x_{1}\right) \widetilde{T}_{n_{2}}\left(x_{2}\right) \cdots \widetilde{T}_{n_{k}}\left(x_{k}\right) \widetilde{T}_{m}(y)$.

The uniqueness and constructability are the most surprising results, since as Buck [1] has shown, $F(x, y)=x y$ has amongst those polynomials of the form

$$
p(x, y)=a_{0}+a_{1}(x+y)+a_{2}\left(x^{2}+y^{2}\right)
$$

infinitely many polynomials of best approximation which are given by:

$$
\alpha f_{1}+\beta f_{2}, \quad \alpha \geqq 0, \quad \beta \geqq 0, \quad \alpha+\beta=1
$$

where

$$
\begin{aligned}
& f_{1}(x, y)=\frac{1}{2}\left(x^{2}+y^{2}\right)-\frac{1}{4}, \\
& f_{2}(x, y)=x+y-\frac{1}{2}\left(x^{2}+y^{2}\right)-\frac{1}{4} .
\end{aligned}
$$

We shall finally normalize the polynomials in a different way and show by construction, the existence of a polynomial, of best approximation in this class. However in this case the question of uniqueness remains open.

1. Notation. Let $n_{1}, n_{2}, \cdots, n_{k}$ be positive fixed integers. Let $\sigma$ be the finite set of vectors $\left\{\left(x_{1 j_{1}}, x_{2 j_{2}}, \cdots, x_{k j_{k}}\right)\right\}$, where $j_{1}, j_{2}, \cdots, j_{k}$ are integers with $0 \leqq j_{1} \leqq n_{1}, 0 \leqq j_{2} \leqq n_{2}, \cdots, 0 \leqq j_{k} \leqq n_{k}$; and where also $-1 \leqq x_{1 j_{1}} \leqq 1,-1 \leqq x_{2 j_{2}} \leqq 1, \cdots,-1 \leqq x_{k j_{k}} \leqq 1$ and no two of the $x_{1 j_{1}}$ are the same, no two of the $x_{2 j_{2}}$ are the same, $\cdots$, no two of the $x_{k j_{k}}$ are the same. Let $Q(x, y)=Q\left(x_{1}, x_{2}, \cdots, x_{k}, y\right)$ be any polynomial in $x_{1}, x_{2}, \cdots, x_{k}$ and $y$ of degree $\leqq n_{1}+n_{2}+\cdots+n_{k}+m-1$ where $Q$ is of degree $\leqq n_{s}$ in $x_{s}, s=1,2, \cdots, k$ and of degree $\leqq m$ in $y$. Let $\pi$ be the set of all such polynomials. Thus if $Q(x, y)$ is in $\pi$

$$
Q(x, y)=p_{m}(x) y^{m}+p_{m-1}(x) y^{m-1}+\cdots+p_{0}(x)
$$

where $p_{m}(x)$ is a polynomial in $x_{1}, x_{2}, \cdots, x_{k}$ of

$$
\text { degree } \leqq n_{1}+n_{2}+\cdots+n_{k}-1
$$

and $p_{s}(x), 0 \leqq s \leqq m-1$, are polynomials of degree $\leqq n_{1}+n_{2}+\cdots+n_{k}$ in $x_{1}, x_{2}, \cdots, x_{k}$. Let

$$
A\left[p_{m} ; \pi, \sigma\right]=\min _{x \text { in } \sigma}\left|x_{1}^{n_{1}} x_{1}^{n_{2}} \cdots x_{k}^{n_{k}}-p_{m}\left(x_{1}, x_{2}, \cdots, x_{k}\right)\right|
$$

which does not depend on the particular $Q$, but only on the class $\pi$ and the leading coefficient polynomial of $y$. 
THEOREM 1. If $Q(x, y)$ is any polynomial in $\pi$ and if $\sigma$ is any set of the type described above then

$$
\max _{\substack{-1 \leq x_{s} \leq 1 \\-1 \leqq y \leqq 1}}\left|x_{1}^{n_{1}} x_{2}^{n_{2}} \cdots x_{k}^{n_{k}} y^{m}-Q\left(x_{1}, x_{2}, \cdots, x_{k}, y\right)\right| \geqq A\left[p_{m} ; \pi, \sigma\right] 2^{1-m} .
$$

Proof. Assume not. Then there exists a $Q^{*}(x, y)$ in $\pi$ and a set $\sigma$ of the type described such that:

$$
\max _{\substack{-1 \leq x_{s} \leq 1 \\-1 \leqq y \leqq 1}}\left|x_{1}^{n_{1}} x_{2}^{n_{2}} \cdots x_{k}^{n_{k}} y^{m}-Q^{*}(x, y)\right|<A\left[p_{m} ; \pi, \sigma\right] 2^{1-m}
$$

consider the polynomial:

$$
\begin{aligned}
P(x, y)= & x_{1}^{n_{1}} x_{2}^{n_{2}} \cdots x_{k}^{n_{k}} y^{m} \\
& -Q^{*}(x, y)-\left[x_{1}^{n_{1}} x_{2}^{n_{2}} \cdots x_{k}^{n_{k}}-p_{m}(x)\right] \widetilde{T}_{m}(y)
\end{aligned}
$$

where $p_{m}(x)$ is the coefficient of $y^{m}$ in $Q^{*}(x, y)$ and where

$$
\widetilde{T}_{m}(y)=2^{1-m} T_{m}(y)=2^{1-m} \cos [m \operatorname{arc} \cos y] .
$$

Then $P(x, y)$ is a polynomial of degree $\leqq m-1$ in $y$ and thus can be written:

$$
P(x, y)=q_{m-1}(x) y^{m-1}+q_{m-2}(x) y^{m-2}+\cdots+q_{0}(x)
$$

where $q_{s}(x), 0 \leqq s \leqq m-1$, are polynomials in $x_{1}, x_{2}, \cdots, x_{k}$ of degree $\leqq n_{1}+n_{2}+\cdots+n_{k}$.

Let $\left(x_{1 j_{1}}, x_{2 j_{2}}, \cdots, x_{k j_{k}}\right)$ belong to $\sigma$ and $y_{r}$ be one of the points

$$
y_{r}=\cos \frac{r \pi}{m}, \quad 0 \leqq r \leqq m, \quad r=\text { integer } .
$$

Then $\widetilde{T}_{m}\left(y_{r}\right)=(-1)^{r} 2^{1-m}$ and we can show that the sign of

$$
P\left[x_{1 j_{1}}, x_{2 j_{2}}, \cdots, x_{k j_{k}}, y_{r}\right]
$$

is the same as the sign of $-\left[x_{1 j_{1}}^{n_{1}} \cdots x_{k j_{k}}^{n_{k}}-p_{m}\left(y_{1 j_{1}}, \cdots, x_{k j_{k}}\right)\right] . \widetilde{T}_{m}\left(y_{r}\right)$. To see this note that:

$$
\begin{aligned}
& \left|\widetilde{T}_{m}\left(y_{r}\right)\right|\left|x_{1 j_{1}}^{n_{1}} \cdots x_{k j_{k}}^{n_{k}}-p_{m}\left(x_{1 j_{1}}, \cdots, x_{k j_{k}}\right)\right| \\
& \quad=\left|x_{1 j_{1}}^{n_{1}} \cdots x_{k j_{k}}^{n_{k}}-p_{m}\left(x_{1 j_{1}}, \cdots, x_{1 j_{k}}\right)\right| 2^{1-m} \\
& \quad \geqq A\left[p_{m} ; \pi, \sigma\right] 2^{1-m} .
\end{aligned}
$$

But by the assumption

$$
\max _{\substack{-1 \leq x_{\leq} \leq 1 \\-1 \leqq y \leqq 1}}\left|x_{1}^{n_{1}} \cdots x_{k}^{n_{k}} y^{m}-Q^{*}(x, y)\right|<A\left[p_{m} ; \pi, \sigma\right] 2^{1-m}
$$

: and thus a fortiori 


$$
\left|x_{1 j_{1}}^{n_{1}} \cdots x_{k_{k}}^{n_{k}} y_{r}^{m}-Q^{*}\left(x_{1 j_{1}}, \cdots, x_{k j_{k}}, y_{r}\right)\right|<A\left[p_{m} ; \pi, \sigma\right] 2^{1-m} .
$$

If we fix $x$ in $\sigma$ then $P(x, y)$ is a polynomial of the one variable $y$ and of degree $\leqq m-1$. And as $y$ takes on the values $y_{r}=\cos (\pi r / m)$, $P(x, y)$ changes sign $m+1$ times. Thus $P(x, y)$ has $m$ zeros, which means $q_{m-1}(x)=0, q_{m-2}(x)=0, \cdots, q_{0}(x)=0$ since $P(x, y)$ is only of degree $\leqq m-1$.

Since $x$ was an arbitrary point of $\sigma$, then

$$
q_{s}\left[x_{1 j_{1}}, x_{2 j_{2}}, \cdots, x_{k j_{k}}\right]=0,0 \leqq s \leqq m-1
$$

where $0 \leqq j_{1} \leqq n_{1}, 0 \leqq j_{2} \leqq n_{2}, \cdots, 0 \leqq j_{k} \leqq n_{k}$. But $q_{s}(x)$ is a polynomial of degree $\leqq n_{1}$ in $x_{1}$, of degree $\leqq n_{2}$ in $x_{2}, \cdots$, of degree $\leqq n_{k}$ in $x_{k}$ and thus

$$
q_{s}\left[x_{1}, x_{2}, \cdots, x_{k}\right] \equiv 0, \quad 0 \leqq s \leqq m-1 .
$$

From which we see $P(x, y) \equiv 0$ and thus:

$$
x_{1}^{n_{1}} \cdots x_{k}^{n} y^{m}-Q^{*}(x, y) \equiv\left[x_{1}^{n_{1}} \cdots x_{k}^{n_{k}}-p_{m}(x)\right] \widetilde{T}_{m}(y) .
$$

But clearly:

$$
\max _{\substack{-1 \leq x_{s} \leq 1 \\-1 \leqq y \leqq 1}}\left|x_{1}^{n_{1}} \cdots x_{k}^{n_{k}}-p_{m}(x)\right||\widetilde{T}(y)| \geqq A\left[p_{m} ; \pi, \sigma\right] 2^{1-m}
$$

which is a contradiction and thus the theorem is proved.

Let us now consider the subset of polynomials $\pi_{0}$ of $\pi$ for which $Q(x, y)$ belongs to $\pi$ and $p_{m}(x)=0$. Then by the above theorem, a. lower bound for the maximum is

$$
A[0 ; \pi, \sigma]=\min _{x \text { in } \sigma}\left|x_{1}^{n_{1}} \cdots x_{k}^{n_{k}}\right|<1
$$

which clearly depends on the set $\sigma$. We shall next show that for this subset $\pi_{0}$, we get a lower bound for the maximum that is independent of $\sigma$ and moreover the lower bound is larger than $A[0 ; \pi, \sigma]$ for all $\sigma$, namely it is unity. In the third theorem we shall show that unity is the best possible lower bound i.e. there is a polynomial in $\pi_{0}$ for which the maximum is $2^{1-m}$.

THEOREM 2. Let $Q(x, y)$ be any polynomial in $\pi_{0}$, then

$$
\max _{\substack{-1 \leq x_{\leq} \leq 1 \\-1 \leqq y \leq 1}} \mid x_{1}^{n_{1}} x_{2}^{n_{2}} \cdots x_{k}^{n} k y^{m}-Q\left(x_{1}, x_{2}, \cdots, x_{k}, y \mid \geqq 2^{1-m} .\right.
$$

Proof. By contradiction. Assume there exists a $Q\left(x_{1}, \cdots, x_{k}, y\right)$ in $\pi_{0}$ such that:

$$
\max _{\substack{-1 \leq x_{s} \leq 1 \\-1 \leqq y \leqq 1}}\left|x_{1}^{n_{1}} x_{2}^{n_{2}} \cdots x_{k}^{n_{k}} y^{m}-Q\left(x_{1}, \cdots, x_{k}, y\right)\right|<2^{1-m} .
$$


Then there exist $\delta_{s}$ 's, $1 \leqq s \leqq k, 1>\delta_{s}>0$ such that:

$$
\max _{\substack{-1 \leqq x \\-1 \leqq y \leqq 1}}\left|x_{1}^{n_{1}} \cdots x_{k}^{n} k y^{m}-Q\left(x_{1}, \cdots, x_{k}, y\right)\right|<2^{1-m} \prod_{s=1}^{k} \delta_{s}^{n_{s}} .
$$

Let $\widetilde{T}_{m}(y)$ be given by (1) and consider the polynomial

$$
P\left(x_{1}, \cdots, x_{k}, y\right) \equiv x_{1}^{n_{1}} \cdots x_{k}^{n} k y^{m}-Q\left(x_{1}, \cdots, x_{k}, y\right)-x_{1}^{n_{1}} \cdots x_{k}^{n_{k}} \widetilde{T}_{m}(y) .
$$

$P\left(x_{1}, \cdots, x_{k}, y\right)$ is a polynomial of degree $\leqq m-1$ in $y$ and of degree $\leqq n_{s}$ in $x_{s} 1 \leqq s \leqq k$.

Let $\sigma^{*}=\left\{\left(x_{1 j_{1}}, x_{2 j_{2}}, \cdots, x_{k j_{k}}\right)\right\}$ where $j_{1}, \cdots, j_{k}$ are integers with

$$
\begin{aligned}
0 \leqq & j_{1} \leqq n_{1}+1,0 \leqq j_{2} \leqq n_{2}+1, \cdots, 0 \leqq j_{k} \leqq n_{k}+1 ; \\
& \delta_{1}<x_{1 j_{1}} \leqq 1, \delta_{2}<x_{2 j_{2}} \leqq 1, \cdots, \delta_{k}<x_{k j_{k}} \leqq 1
\end{aligned}
$$

and the $x_{1 j_{1}}$ are distinct, $\cdots$, the $x_{k j_{k}}$ are distinct.

Note that for $x$ in $\sigma^{*}$, the sign of $P\left(x_{1 j_{1}}, \cdots, x_{k j_{k}}, y\right)$ is the same as the sign of $-x_{1 j_{1}}^{n_{1}} \cdots x_{k n_{k}}^{n_{k}} \widetilde{T}_{m}\left(y_{r}\right)$ for $y_{r}=\cos (r \pi / m), r=0,1, \cdots, m$. This follows from the fact that:

$$
\left|x_{1_{1}}^{n_{1}} \cdots x_{k j_{k}}^{n_{k}} y_{r}^{m}-Q\left(x_{1}, \cdots, x_{k}, y_{r}\right)\right|<2^{1-m} \prod_{s=1}^{k} \delta_{s}^{n_{s}}
$$

and the fact that:

$$
\left|x_{1_{1}}^{n_{1}} \cdots x_{k j_{k}}^{n_{k}} \widetilde{T}_{m}\left(y_{r}\right)\right|=2^{1-m} \prod_{s=1}^{k} x_{s_{s}}^{n_{s}}>2^{1-m} \prod_{s=1}^{k} \delta_{s}^{n_{s}} .
$$

Thus we conclude that $P\left(x_{1 j_{1}}, \cdots, x_{k j_{k}}, y\right)$ has $m+1$ sign changes for $\left(x_{1 j_{1}}, \cdots, x_{k j_{k}}\right)$ in $\sigma^{*}$. Let us write

$$
P(x, y)=p_{m-1}(x) y^{m-1}+p_{m-2}(x) y^{m-2}+\cdots+p_{0}(x)
$$

where $p_{s}(x), 0 \leqq s \leqq m-1$, are polynomials of degree $\leqq n_{s}$ in $x_{s}, 0 \leqq s \leqq k$. For each $x$ in $\sigma^{*}, P(x, y)$ has $m+1$ sign changes and thus $p_{m-1}(x)=0$, $p_{m-2}(x)=0, \cdots, p_{0}(x)=0$ for each $x$ in $\sigma^{*}$. If for $\left(x_{1 j_{1}}, x_{2 j_{2}}, \cdots, x_{k j_{k}}\right)$ in $\sigma^{*}$, we fix all but the first component, we get $n_{1}+2$ values in $\sigma^{*}$ for which $p_{s}(x)=0,0 \leqq s \leqq m-1$, but these $p_{s}(x)$ are of degree $\leqq n_{1}$ in $x_{1}$ and thus $p_{s}\left(x_{1}, x_{2 j_{2}}, x_{3 j_{3}}, \cdots, x_{k j_{k}}\right)=0$ for all real $x_{1}$. Continuing. in this way, we see that $p_{s}\left(x_{1}, x_{2}, \cdots, x_{k}\right) \equiv 0$ for all $\left(x_{1}, x_{2}, \cdots, x_{k}\right), x_{s}$ real. Thus:

$$
P\left(x_{1}, x_{2}, \cdots, x_{k}, y\right) \equiv 0
$$

for all real $x_{s}$ and real $y$. Thus

$$
x_{1}^{n_{1}} \cdots x_{k}^{n_{k}} \widetilde{T}_{m}(y) \equiv x_{1}^{n_{1}} \cdots x_{k}^{n} k y^{m}-Q\left(x_{1}, \cdots, x_{k}, y\right) .
$$

But 


$$
\max _{\substack{-1 \leq x \leq 1 \\-1 \leqq y \leqq 1}}\left|x_{1}^{n_{1}} \cdots x_{k}^{n_{k}} \widetilde{T}_{m}(y)\right|=2^{1-m}
$$

which gives a contradiction and the theorem is proved.

2. Normalization of competing polynomials and construction of the best polynomial. We shall now consider a subset $\pi(\beta)$ of the set of polynomials $\pi$. We shall then answer the question of existence, uniqueness and constructability of the best polynomial approximation in the maximum norm to zero within this class $\pi(\beta)$ on the cube

$$
-1 \leqq x_{1} \leqq 1, \cdots,-1 \leqq x_{k} \leqq 1,-1 \leqq y \leqq 1 .
$$

It is apparent from Theorem 1, that if we want uniqueness independent of $\sigma$, it is necessary to consider some subset of $\pi$.

Definition. A polynomial

$$
\begin{aligned}
Q(x, y)= & p_{m}\left(x_{1}, x_{2}, \cdots, x_{k}\right) y^{m} \\
& +p_{m-1}\left(x_{1}, x_{2}, \cdots, x_{k}\right) y^{m-1}+\cdots+p_{0}\left(x_{1}, x_{2}, \cdots, x_{k}\right)
\end{aligned}
$$

which is in $\pi$ and for which

$$
x_{1}^{n_{1}} x_{2}^{n_{2}} \cdots x_{k}^{n} k-p_{m}\left(x_{1}, x_{2} \cdots x_{k}\right)=\widetilde{T}_{n_{1}}\left(x_{1}\right) \widetilde{T}_{n_{2}}\left(x_{2}\right) \cdots \widetilde{T}_{n_{k}}\left(x_{k}\right)
$$

is said to be in $\pi(\beta)$.

Lemma. Let $q(y)$ be a polynomial in $y$, let $y_{0}>y_{1}>\cdots>y_{m}$ be any set of real numbers for which

$$
q\left(y_{0}\right) \leqq 0, q\left(y_{1}\right) \geqq 0, q\left(y_{2}\right) \leqq 0, \cdots(-1)^{m} q\left(y_{m}\right) \leqq 0 .
$$

Then $q(y)$ has $m$ zeros including multiplicities on $\left[y_{0}, y_{m}\right]$.

Proof. (by induction): For $m=1$ obvious. Assume theorem to. be true for $m \leqq k$. Let $y_{0}>y_{1}>y_{2}>\cdots>y_{k+1}$ be any set of real numbers such that

$$
q\left(y_{0}\right) \leqq 0, q\left(y_{1}\right) \geqq 0, \cdots(-1)^{k} q\left(y_{k}\right) \leqq 0,(-1)^{k+1} q\left(y_{k+1}\right) \leqq 0 .
$$

Case 1. $q\left(y_{s}\right) \neq 0$ for some $1 \leqq s \leqq k$. Then by the induction hypothesis $q(y)$ has $s$ zeros on $\left[y_{0}, y_{s}\right]$ and has $k+1-s$ zeros on $\left[y_{s}, y_{k+1}\right]$. But $q\left(y_{s}\right) \neq 0$ thus $q(y)$ has $s$ zeros on $y_{0} \leqq y \leqq y_{s}$ and thus $q(y)$ has $s+(k+1-s)=k+1$ zeros on $\left[y_{0}, y_{k+1}\right]$.

Case 2. $q\left(y_{0}\right)<0$. Then unless $q\left(y_{s}\right)=0$ for $1 \leqq s \leqq k$ we are in Case 1 and we are finished. Therefore, assume $q\left(y_{s}\right)=0,1 \leqq s \leqq k$. 
We may as well assume $q(y)<0$ on $\left(y_{0}, y_{1}\right)$ since if not then $q(y)$ has a zero there because $q\left(y_{0}\right)<0$, and we are finished. Also, we may as well assume $q(y)>0$ on $\left(y_{1}, y_{2}\right)$ since if not and $q(y)$ has no zeros on $\left(y_{1}, y_{2}\right)$ (if does have a zero then we are finished) then since $q\left(y_{0}\right)<0$ and $q\left(y_{1}\right)=0$, we must have that $q(y)$ has 2 zeros in $\left(y_{0}, y_{2}\right)$, continuing in this way we see that we may as well assume that $(-1)^{s} q(y)<0$ on $\left(y_{s}, y_{s+1}\right)$ for $0 \leqq s \leqq k$. In particular $(-1)^{k} q(y)<0$ for $y$ on $\left(y_{k}, y_{k+1}\right)$. But by assumption $(-1)^{k+1} q\left(y_{k+1}\right) \leqq 0$. Thus by the continuity of $q(y)$, we have $q\left(y_{k+1}\right)=0$ and $q\left(y_{s}\right)=0$ for $1 \leqq s \leqq k+1$ i.e. $q(y)$ has $k+1$ zeros on $\left[y_{0}, y_{k+1}\right]$.

Case 3. $q\left(y_{0}\right)=0$ proof is obvious making use of Case 1.

THEOREM 3. There exists a unique $Q^{*}(x, y)$ in $\pi(\beta)$ such that

$$
\max _{\substack{-1 \leq x_{s} \leq 1 \\-1 \leqq y \leqq 1}}\left|x_{1}^{n_{1}} x_{2}^{n_{2}} \cdots x_{k}^{n} k y^{m}-Q^{*}(x, y)\right|
$$

is a minimum. Moreover:

$$
Q^{*}(x, y)=-\widetilde{T}_{n_{1}}\left(x_{1}\right) \widetilde{T}_{n_{2}}\left(x_{2}\right) \cdots \widetilde{T}_{n_{k}}\left(x_{k}\right) \widetilde{T}_{m}(y)+x_{1}^{n_{1}} x_{2}^{n_{2}} \cdots x_{k}^{n} y^{m} .
$$

Proof. Existence by construction. Let the $\sigma$ of Theorem 1 be the special set of vectors

$$
\sigma(\beta)=\left\{\left(x_{1 j_{1}}, x_{2 j_{2}}, \cdots, x_{k j_{k}}\right)\right\}
$$

where

$$
\begin{aligned}
& x_{1 j_{1}}=\cos \left(j_{1} \pi / n_{1}\right), x_{2 j_{2}}, \cdots, x_{k j_{k}}=\cos \left(j_{k} \pi / n_{k}\right) \\
& 0 \leqq j_{1} \leqq n_{1}, 0 \leqq j_{2} \leqq n_{2}, \cdots, 0 \leqq j_{k} \leqq n_{k}
\end{aligned}
$$

Then

$$
\begin{aligned}
A\left[p_{m}, \pi(\beta), \sigma(\beta)\right] & =\min _{x \text { in } \sigma(\beta)}\left|x_{1}^{n_{1}} x_{2}^{n_{2}} \cdots x_{k}^{n_{k}}-p_{m}\left(x_{1}, x_{2}, \cdots, x_{k}\right)\right| \\
& =\min _{x \text { in } \sigma(\beta)}\left|\widetilde{T}_{n_{1}}\left(x_{1}\right) \widetilde{T}_{n_{2}}\left(x_{1}\right) \cdots \widetilde{T}_{n_{k}}\left(x_{k}\right)\right| \\
& =2^{1-n_{1}} 2^{1-n_{2}} \cdots 2^{1-n_{k}} .
\end{aligned}
$$

Thus by Theorem 1

$$
\max _{\substack{-1 \leqq x_{j} \leqq 1 \\-1 \leqq y \leqq 1}}\left|x_{1}^{n_{1}} x_{2}^{n_{2}} \cdots x_{k}^{n_{k}} y^{m}-Q(x, y)\right| \geqq 2^{1-n_{1}} 2^{1-n_{2}} \cdots 2^{1-n_{k}} 2^{1-m} .
$$

But the polynomial

$$
Q^{*}(x, y)=x_{1}^{n_{1}} x_{2}^{n_{2}} \cdots x_{k}^{n_{k}} y^{m}-\widetilde{T}_{n_{1}}\left(x_{1}\right) \widetilde{T}_{n_{2}}\left(x_{2}\right) \cdots \widetilde{T}_{n_{k}}\left(x_{k}\right) \widetilde{T}_{m}(y)
$$


clearly belongs to $\pi(\beta)$ and

$$
\max _{\substack{-1 \leq x_{s} \leq 1 \\-1 \leqq y \leqq 1}}\left|x_{1}^{n_{1}} x_{2}^{n_{2}} \cdots x_{k}^{n} k y^{m}-Q^{*}(x, y)\right|=2^{1-n_{1}} 2^{1-n_{2}} \cdots 2^{1-n_{k}} 2^{1-m} .
$$

Thus $Q^{*}(x, y)$ is a best approximation from the set $\pi(\beta)$

Uniqueness. Let $Q^{*}(x, y)$ in $\pi(\beta)$ be a polynomial of best approximation and let

$$
\begin{aligned}
P(x, y)= & x_{1}^{n_{1}} x_{2}^{n_{2}} \cdots x_{k}^{n_{k}} y^{m}-Q^{*}(x, y)-\widetilde{T}_{n_{1}}\left(x_{\mathrm{i}}\right) \cdots \widetilde{T}_{n_{k}}\left(x_{k}\right) \widetilde{T}_{m}(y) \\
= & {\left[x_{1}^{n_{1}} x_{2}^{n_{1}} \cdots x_{k}^{n_{k}}-p_{m}(x)\right] y^{m}-p_{m-1}(x) y^{m-1}-\cdots p_{0}(x) } \\
& -\widetilde{T}_{n_{1}}\left(x_{1}\right) \widetilde{T}_{n_{2}}\left(x_{2}\right) \cdots \widetilde{T}_{n_{k}}\left(x_{k}\right) \widetilde{T}_{m}(y) \\
= & q_{m-1}(x) y^{m-1}+q_{m-2}(x) y^{m-2}+\cdots+q_{0}(x)
\end{aligned}
$$

where $q_{m-1}(x), \cdots, q_{0}(x)$ are polynomials of degree $\leqq n_{s}$ in $x_{s} 0 \leqq s \leqq k$ since $Q^{*}(x, y)$ is in $\pi(\beta)$.

Let $x^{*}=\left(x_{1}^{*}, x_{2}^{*}, \cdots, x_{k}^{*}\right)$ be a fixed but arbitrary element of $\sigma(\beta)$. Then we claim that $P\left(x^{*}, y\right)$ has $m$ zeros including multiplicities in $[-1,1]$. To see this let $y_{s}=\cos (s \pi / m), 0 \leqq s \leqq m$, then since

$$
\begin{aligned}
& \left|x_{1}^{* n_{1}} x_{2}^{* n_{2}} \cdots x_{k}^{* n_{k}} y^{m}-Q^{*}\left(x^{*}, y\right)\right| \leqq 2^{1-n_{1}} 2^{1-n_{2}} \cdots 2^{1-n_{k}} 2^{1-m}, \\
& P\left(x^{*}, y_{0}\right) \leqq 0, P\left(x^{*}, y_{1}\right) \geqq 0, \cdots(-1)^{m} P\left(x^{*}, y_{m}\right) \leqq 0 .
\end{aligned}
$$

By the lemma $P\left(x^{*}, y\right)$ has $m$ zeros counting multiplicities for $-1 \leqq y \leqq 1$.

Thus $P\left(x^{*}, y\right)$ has $m$ zeros but is only a polynomial of degree $m-1$, thus $P\left(x^{*}, y\right) \equiv 0$. But this holds for all $x^{*}$ in $\sigma(\beta)$, thus $P(x, y) \equiv 0$ and the theorem is proved.

We could formulate Theorem 3 in the following way. Let $\pi(k)$, $k \geqq 1$, be the set of polynomials of the form

$$
Q(x, y)=p_{m}\left(x_{1}, \cdots, x_{k}\right) x_{k+1}^{m}+p_{m-1}(x) x_{k+1}^{m-1}+\cdots+p_{0}(x)
$$

which is of degree $\leqq n_{s}$ in $x_{s}, 1 \leqq s \leqq k$ and for which $p_{m}\left(x_{1} \cdots x_{k}\right)$ is a polynomial that best approximates zero, if such exists, on the cube $I_{1} \times I_{2} \times \cdots \times I_{k}, I_{s}=[-1,1], 1 \leqq s \leqq k$.

Theorem 3 alternate. For $k=2,3,4 \cdots$, the following is true:

Statement k. $\pi(k-1)$ is not empty and there exists a unique $M_{k}\left(x_{1}, x_{2}, \cdots, x_{k}, x_{k+1}\right)$ in $\pi(k)$ such that:

$$
\max _{\substack{-1 \leq x_{s} \leq 1 \\-1 \leqq y \leqq 1}}\left|M_{k}\left(x_{1}, x_{2}, \cdots, x_{k}, x_{k+1}\right)\right|
$$

is a minimum. Moreover: 


$$
M_{k}\left(x_{1}, x_{2}, \cdots, x_{k}, x_{k+1}\right)=\widetilde{T}_{n_{1}}\left(x_{1}\right) \widetilde{T}_{n_{2}}\left(x_{2}\right) \cdots \widetilde{T}_{n_{k}}\left(x_{k}\right) \widetilde{T}_{n_{k+1}}\left(x_{k_{n+1}}\right) .
$$

Proof. Obvious.

Finally we wish to prove:

THEOREM 4. There exists a monic polynomial

$$
P\left(x_{1}, \cdots, x_{k}, y\right)=x_{1}^{n_{1}} \cdots x_{k}^{n_{k}} y^{m}-Q\left(x_{1}, \cdots, x_{k}, y\right)
$$

where $Q(x, y)$ belongs to $\pi_{0}$ that best approximates zero on the cube $I_{1} \times I_{2} \times \cdots \times I_{k+1}, I_{s}=[-1,1]$. The polynomial is

$$
x_{1}^{n_{1}} \cdots x_{k}^{n_{k}} \widetilde{T}_{m}(y) \text {. }
$$

Proof. By Theorem 2

$$
\max _{\substack{-1 \leqq x_{s} \leq 1 \\-1 \leqq y \leqq 1}}\left|P\left(x_{1}, \cdots, x_{k}^{n} k, y\right)\right| \geqq 2^{1-m} .
$$

But $x_{1}^{n_{1}} \cdots x_{k}^{n_{k}} \widetilde{T}_{m}(y)$ is a monic polynomial of the correct form with

$$
\max _{\substack{-1 \leq x_{s} \leq 1 \\-1 \leqq y \leqq 1}}\left|x_{1}^{n_{1}} \cdots x_{k}^{n} k \widetilde{T}_{m}(y)\right|=2^{1-m} .
$$

Thus the theorem is correct.

The question of uniqueness in this case is an open one.

\section{REFERENCE}

1. R. C. Buck, Linear spaces and approximation theory, On numerical approximation, Edited by R. E. Langer, Published by the University of Wisconsin Press, 1959.

University of California, Santa Barbara 



\section{PACIFIC JOURNAL OF MATHEMATICS}

\section{EDITORS}

H. Samelson

Stanford University

Stanford, California

R. M. Blumenthal

University of Washington

Seattle, Washington 98105
J. Dugundu

University of Southern California Los Angeles, California 90007

*Richard Arens

University of California

Los Angeles, California 90024

\section{ASSOCIATE EDITORS}
E. F. BECKENBACH
B. H. NeumanN
F. WOLF
K. YOSIDA

\section{SUPPORTING INSTITUTIONS}

UNIVERSITY OF BRITISH COLUMBIA CALIFORNIA INSTITUTE OF TECHNOLOGY UNIVERSITY OF CALIFORNIA MONTANA STATE UNIVERSITY

UNIVERSITY OF NEVADA

NEW MEXICO STATE UNIVERSITY

OREGON STATE UNIVERSITY

UNIVERSITY OF OREGON

OSAKA UNIVERSITY

UNIVERSITY OF SOUTHERN CALIFORNIA
STANFORD UNIVERSITY

UNIVERSITY OF TOKYO

UNIVERSITY OF UTAH

WASHINGTON STATE UNIVERSITY

UNIVERSITY OF WASHINGTON

AMERICAN MATHEMATICAL SOCIETY CALIFORNIA RESEARCH CORPORATION SPACE TECHNOLOGY LABORATORIES NAVAL ORDNANCE TEST STATION 


\section{Pacific Journal of Mathematics}

\section{Vol. 15, No. $1 \quad$ September, 1965}

Donald Charles Benson, Unimodular solutions of infinite systems of linear

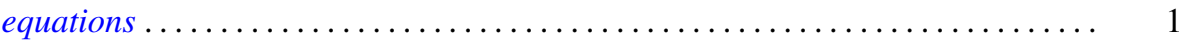

Richard Earl Block, Transitive groups of collineations on certain designs . . . . . . 13

Barry William Boehm, Existence of best rational Tchebycheff approximations .... . 19

Joseph Patrick Brannen, A note on Hausdorff's summation methods . . . . . . . . . . 29

Dennison Robert Brown, Topological semilattices on the two-cell ............ 35

Peter Southcott Bullen, Some inequalities for symmetric means . . . . . . . . . . 47

David Geoffrey Cantor, On arithmetic properties of coefficients of rational

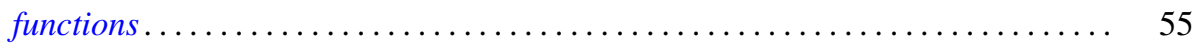

Luther Elic Claborn, Dedekind domains and rings of quotients . . . . . . . . . 59

Allan Clark, Homotopy commutativity and the Moore spectral sequence ........ 65

Allen Devinatz, The asymptotic nature of the solutions of certain linear systems of

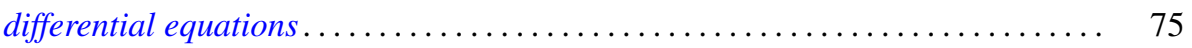

Robert E. Edwards, Approximation by convolutions ................... 85

Theodore William Gamelin, Decomposition theorems for Fredholm operators . . . . . 97

Edmond E. Granirer, On the invariant mean on topological semigroups and on

topological groups .................................. 107

Noel Justin Hicks, Closed vector fields . . . . . . . . . . . . . . . . . . . 141

Charles Ray Hobby and Ronald Pyke, Doubly stochastic operators obtained from

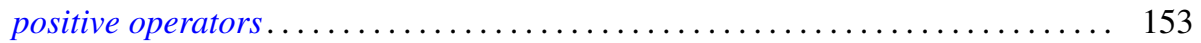

Robert Franklin Jolly, Concerning periodic subadditive functions . . . . . . . . . 159

Tosio Kato, Wave operators and unitary equivalence . . . . . . . . . . . . . . 171

Paul Katz and Ernst Gabor Straus, Infinite sums in algebraic structures . . . . . . . 181

Herbert Frederick Kreimer, Jr., On an extension of the Picard-Vessiot theory ...... 191

Radha Govinda Laha and Eugene Lukacs, On a linear form whose distribution is

identical with that of a monomial ......................... 207

Donald A. Ludwig, Singularities of superpositions of distributions . . . . . . . . . 215

Albert W. Marshall and Ingram Olkin, Norms and inequalities for condition

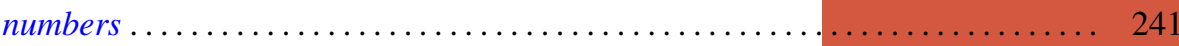

Horace Yomishi Mochizuki, Finitistic global dimension for rings . . . . . . . . . . 249

Robert Harvey Oehmke and Reuben Sandler, The collineation groups of division

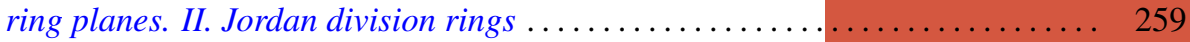

George H. Orland, On non-convex polyhedral surfaces in $E^{3} \ldots \ldots \ldots \ldots \ldots \ldots \ldots 267$

Theodore G. Ostrom, Collineation groups of semi-translation planes . . . . . . . . 273

Arthur Argyle Sagle, On anti-commutative algebras and general Lie triple

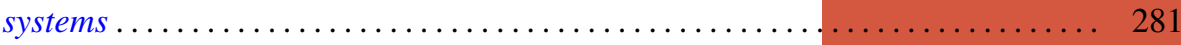

Laurent Siebenmann, A characterization of free projective planes . . . . . . . . . 293

Edward Silverman, Simple areas.................................. 299

James McLean Sloss, Chebyshev approximation to zero .................. 305

Robert S. Strichartz, Isometric isomorphisms of measure algebras . . . . . . . . . 315

Richard Joseph Turyn, Character sums and difference sets . . . . . . . . . . . . 319

L. E. Ward, Concerning Koch's theorem on the existence of arcs . . . . . . . . . . 347

Israel Zuckerman, A new measure of a partial differential field extension ......... 357 